\title{
Finite Galois covers, cohomology jump loci, formality properties, and multinets
}

\author{
Alexandru Dimca And Stefan Papadima
}

\begin{abstract}
We explore the relation between cohomology jump loci in a finite Galois cover, formality properties and algebraic monodromy action. We show that the jump loci of the base and total space are essentially the same, provided the base space is 1 -formal and the monodromy action in degree 1 is trivial. We use reduced multinet structures on line arrangements to construct components of the first characteristic variety of the Milnor fiber in degree 1, and to prove that the monodromy action is non-trivial in degree 1. For an arbitrary line arrangement, we prove that the triviality of the monodromy in degree 1 can be detected in a precise way, by resonance varieties.
\end{abstract}

Mathematics Subject Classification (2010): 32S22 (primary); 52C30 55N25, 55P62 (secondary).

\section{Introduction and statement of results}

A homogeneous, degree $d$ polynomial $Q \in \mathbb{C}\left[x_{0}, \ldots, x_{n}\right]$ defines a hypersurface in $\mathbb{P}^{n}, V=V(Q)=\{Q=0\}$, its complement $M=M(Q)$, the Milnor fiber in $\mathbb{C}^{n+1}, F=F(Q)=\{Q=1\}$, and a $d$-fold cyclic Galois cover, $p: F \rightarrow M$, with geometric monodromy $h: F \rightarrow F$. In spite of the abundance of available algebrogeometric and topological methods, the analysis of the algebraic monodromy action (over $\mathbb{C}$ ), $h^{*}: H^{*}(F) \rightarrow H^{*}(F)$, remains a challenge, as documented by an extensive literature. Even when $Q$ completely decomposes into distinct linear factors, the additional combinatorial tools from the theory of hyperplane arrangements have not succeeded to elucidate the subject.

Given a connected, finite CW-complex $X$, one may consider its associated characteristic varieties $\mathcal{V}_{m}^{i}(X)$ (alias its Green-Lazarsfeld sets, when $X$ is a smooth projective variety, see [20] for the original setting involving line bundles with trivial Chern class, and [2] for the reformulation in terms of local systems). They are

The first author was partially supported by the French-Romanian Programme LEA Math-Mode and ANR-08-BLAN-0317-02 (SEDIGA). The second author was partially supported by the French-Romanian Programme LEA Math-Mode and CNCSIS ID-1189/2009-2011 (Romania).

Received June 29, 2009; accepted in revised form February 25, 2010. 
defined as jump varieties in degree $i$, for the cohomology of $X$ with coefficients in rank 1 local systems, and live in $\mathbb{T}(X):=\operatorname{Hom}\left(\pi_{1}(X), \mathbb{C}^{*}\right)$. Their 'infinitesimal' approximations, the resonance varieties $\mathcal{R}_{m}^{i}(X)$ associated to the cohomology ring of $X$, are homogeneous subvarieties of $H^{1}(X, \mathbb{C})$. See Section 2 for the precise definitions. Encouraged by recent progress on jump loci, we aim in this note to start their study for Milnor fibers, and relate them to the algebraic monodromy.

\section{1.}

For a 'general' hyperplane arrangement in $\mathbb{P}^{n}$, it is well-known that $h^{*}=$ id in degree $*<n$. See e.g. [8,9], [12, Chapter 6], [22, 24], for various (classes of) examples of this kind. Guided by these examples, we begin our study in Section 2 with jump loci in arbitrary covers, $p: F \rightarrow M$, with finite Galois group $C$, with emphasis on implications of the (partial) triviality of the algebraic monodromy action of $C$ on $H^{*}(F)$.

We start by showing that $p^{*}: \operatorname{Hom}\left(\pi_{1}(M), \mathbb{C}^{*}\right) \rightarrow \operatorname{Hom}\left(\pi_{1}(F), \mathbb{C}^{*}\right)$ sends $\mathcal{V}_{m}^{i}(M)$ into $\mathcal{V}_{m}^{i}(F)$, for all $i$ and $m$. For the rest of Section 2, we fix $q \geq 1$ and suppose that $C$ acts trivially on $H^{*}(F)$, for $* \leq q$. We first note that under this assumption the isomorphism $p^{*}: H^{1}(M, \mathbb{C}) \stackrel{\sim}{\longrightarrow} H^{1}(F, \mathbb{C})$ identifies $\mathcal{R}_{m}^{i}(M)$ with $\mathcal{R}_{m}^{i}(F)$, for all $i \leq q$ and all $m$. Unfortunately, the proof does not work for characteristic varieties.

This difficulty may be bypassed by resorting to a recent result from [18]. This result (recorded here as Theorem 2.7) establishes a natural bijection between the non-translated irreducible components of $\mathcal{V}_{m}^{1}(X)$ (i.e., those containing the trivial local system 1) and the irreducible components of $\mathcal{R}_{m}^{1}(X)$, for all $m$, under a socalled 1-formality hypothesis on the connected CW-complex $X$.

We are thus naturally led to examine partial formality properties in finite Galois covers. Their definition is inspired from D. Sullivan's [35] homotopy theory for commutative differential graded algebras in characteristic zero. To any such DGA, $\left(A^{*}, d_{A}\right)$, one may associate its cohomology algebra $H^{*}(A)$, viewed as a DGA with trivial differential. For a fixed $q \geq 1,\left(A^{*}, d_{A}\right)$ is called $q$-formal if it can be connected to $\left(H^{*}(A), d=0\right)$ by a zigzag of DGA maps, each inducing in cohomology an isomorphism in degree up to $q$, and a monomorphism in degree $q+1$. A connected polyhedron $X$ is called $q$-formal if Sullivan's DGA of PL forms on $X$ is $q$-formal. See Definition 2.3 and Remark 2.4 for another, equivalent, approach to this notion. For $q=\infty$, one recovers Sullivan's celebrated notion of formality, which implies $q$-formality, for all $q$. The 1-formality of $X$ depends only on $\pi_{1}(X)$. See Section 2 for more details.

Interesting examples abound. Hyperplane arrangement complements are formal [5]. If a smooth quasi-projective variety $X$ has vanishing Deligne weight filtration $W_{1} H^{1}(X)$ (which happens $e . g$. for a projective hypersurface complement), then $X$ is 1-formal [25]. In particular, pure braid groups are 1-formal. This was extended to pure welded braid groups in [3]. Finitely generated Artin groups are also 1-formal [21]. Under certain trivial monodromy assumptions, Artin kernels are 
1-formal, too [27].

The interplay between 1-formality and algebraic monodromy in fibrations was recently investigated in [28]. We pursue this approach for finite Galois covers with trivial algebraic monodromy up to degree $q$, proving that $M$ is $q$-formal if and only if $F$ is $q$-formal. Here is our main result from Section 2.

Theorem 1.1 (Theorem 2.8). Let $p: F \rightarrow M$ be a finite Galois cover with group $C$. Assume that $C$ acts trivially on $H^{1}(F)$, and $M$ is 1-formal. Then $p^{*}: \mathbb{T}(M) \rightarrow$ $\mathbb{T}(F)$ gives a natural bijection between non-translated components in $\mathcal{V}_{m}^{1}(M)$ and $\mathcal{V}_{m}^{1}(F)$, for all $m$.

\section{2.}

In Section 3, we specialize our discussion to the Galois cover associated to a line arrangement in $\mathbb{P}^{2}, \mathcal{A}=\left\{L_{1}, \ldots, L_{d}\right\}$, with $L_{i}$ given by the equation $f_{i}=0$. It follows from Theorem 2.7 that the non-translated components of $\mathcal{V}_{m}^{1}(M)$ are in bijection with the components of $\mathcal{R}_{m}^{1}(M)$, for all $m$. At the same time, it is well-known that $\mathcal{R}_{m}^{1}(M)$ depends only on the combinatorics of the arrangement, as follows from basic work by Orlik and Solomon [26]. A nice recent result of Falk and Yuzvinsky [19] gives a combinatorial parametrization for global components of $\mathcal{R}_{1}^{1}(M)$ (i.e., components not coming from a proper subarrangement).

The key combinatorial notion from [19] is that of a multinet supported by $\mathcal{A}$. It involves two data: a multiplicity function $\mu$ on the set $\{1, \ldots, d\}$ with values in $\mathbb{Z}_{>0}$, and a partition of this set, $\mathcal{A}_{1} \cup \ldots \cup \mathcal{A}_{k}$, with at least 3 elements, satisfying certain axioms. The first axiom requires that $\sum_{i \in \mathcal{A}_{j}} \mu(i)=e$, independently of $j$. Starting with a multinet structure on $\mathcal{A}$, the authors of [19] construct an admissible map (in the sense of Arapura [1]), $f: M \rightarrow S$, where $S=\mathbb{P}^{1} \backslash\{k$ points $\}$, and the global component $f^{*}\left(H^{1}(S)\right)$ of $\mathcal{R}_{1}^{1}(M)$, whose corresponding non-translated component of $\mathcal{V}_{1}^{1}(M)$ is $W:=f^{*}(\mathbb{T}(S))$. They also prove that every global component of $\mathcal{R}_{1}^{1}(M)$ arises in this way.

We will say that a multinet is reduced if $\mu$ takes only the value 1 . Classical nets provide reduced examples. There are also situations when $\mathcal{A}$ supports no multinet, simply because there is no global component of $\mathcal{R}_{1}^{1}(M)$; see Example 10.5 from [34]. In a recent preprint [36], Yuzvinsky shows that $k$ is 3 or 4, for any multinet. The only known example with $k=4$ comes from the Hesse pencil.

Recall from Theorem 1.1 that $p^{*}(W)$ is a non-translated component of $\mathcal{V}_{1}^{1}(F)$, if $h^{*}=$ id on $H^{1}(F)$, where $W$ is the non-translated component of $\mathcal{V}_{1}^{1}(M)$ associated to an arbitrary multinet on $\mathcal{A}$. Our second main result in this note is derived in Section 3. It establishes a connection between the existence of reduced multinet structures on a line arrangement, and the triviality of the algebraic monodromy in degree 1 .

Theorem 1.2. Let $W$ be the non-translated component of $\mathcal{V}_{1}^{1}(M)$ associated to a reduced multinet on $\mathcal{A}$. Then there is a non-translated component of $\mathcal{V}_{1}^{1}(F), W^{\prime}$, strictly containing $p^{*}(W)$. In particular, $h^{*} \neq \mathrm{id}$, on $H^{1}(F)$. 
See Theorem 3.1 and Corollary 3.2 for more precise statements. We point out that the existence of a non-reduced multinet is not enough to infer the non-triviality of the monodromy; see Remark 3.4 (ii).

In Section 4, we examine Galois covers associated to plane projective curves, and exploit the mixed Hodge structure. When $p: F \rightarrow M$ comes from an arbitrary line arrangement, we show that $h^{*}=$ id on $H^{1}(F)$ if and only if $p^{*}\left(\mathcal{R}_{1}^{1}(M)\right)=$ $\mathcal{R}_{1}^{1}(F)$, thereby improving Theorem 1.1 in this case. The proof relies on a general result that relates the mixed Hodge structure on $H^{*}(F)$ and the algebraic monodromy action; see Theorem 4.1. Finally, we extend the natural bijection from Theorem 1.1, for arbitrary plane curves. In this situation, we show that, when $h^{*}=$ id on $H^{1}(F), p^{*}$ essentially identifies all components in $\mathcal{V}_{1}^{1}(M)$ and $\mathcal{V}_{1}^{1}(F)$, of dimension at least 2. See Proposition 4.5 for a more precise statement.

In the recent preprint [7], the relation between nets and monodromy of the Milnor fiber of a line arrangement is considered from an alternative viewpoint, see especially [7, Theorem 1].

ACKNOWLEDGEMENTS. Much of this work was done during visits at Université de Nice-Sophia Antipolis by S. Papadima (June 2008 and May 2009). He thanks the Laboratoire Jean A. Dieudonné for its support and hospitality during his stay in Nice, France. Additionally, A. Dimca is grateful to ASSMS, Government College University, Lahore, Pakistan, where part of the work on this paper was done.

\section{Finite Galois covers}

In this section, we begin our analysis of cohomology jump loci in a purely topological context. Let $X$ be a connected CW-complex with finite 1-skeleton, and (finitely generated) fundamental group $G=\pi_{1}(X)$. For a characteristic zero field $\mathbb{K}$, denote by $\mathbb{T}(X, \mathbb{K})=\operatorname{Hom}\left(G, \mathbb{K}^{*}\right)=\operatorname{Hom}\left(G_{\mathrm{ab}}, \mathbb{K}^{*}\right)$ the character torus. (When there is no explicit mention about coefficients, we mean that $\mathbb{K}=\mathbb{C}$.) Clearly, the algebraic group $\mathbb{T}(X, \mathbb{K})$ is the direct product with a finite group of the connected character torus, $\mathbb{T}^{0}(X, \mathbb{K})=\operatorname{Hom}\left(G_{\mathrm{ab}} /\right.$ torsion, $\left.\mathbb{K}^{*}\right)=\left(\mathbb{K}^{*}\right)^{b_{1}(G)}$.

The characteristic varieties

$$
\mathcal{V}_{m}^{i}(X, \mathbb{K})=\left\{\mathcal{L} \in \mathbb{T}(X, \mathbb{K}) \mid \operatorname{dim}_{\mathbb{K}} H^{i}(X, \mathcal{L}) \geq m\right\}
$$

are defined for $i \geq 0$ and $m>0$. If $X$ has finite $q$-skeleton $(q \geq 1)$, they are Zariski closed subsets of the character torus, for $i \leq q$ and $m>0$. Their approximations, the resonance varieties

$$
\mathcal{R}_{m}^{i}(X, \mathbb{K})=\left\{\alpha \in H^{1}(X, \mathbb{K}) \mid \operatorname{dim}_{\mathbb{K}} H^{i}\left(H^{\bullet}(X, \mathbb{K}), \mu_{\alpha}\right) \geq m\right\}
$$

(where $\mu_{\alpha}$ denotes right-multiplication by $\alpha$ in the cohomology ring of $X$ ) are defined for $i \geq 0$ and $m>0$. If $X$ has finite $q$-skeleton $(q \geq 1)$, they are homogeneous, Zariski closed subsets of the affine space $H^{1}(X, \mathbb{K})=\mathbb{K}^{b_{1}(G)}$. We mean 
that $i=1$, whenever the upper index $i$ is missing. In this case, both $\mathcal{V}_{m}(X, \mathbb{K})$ and $\mathcal{R}_{m}(X, \mathbb{K})$ depend only on $G$, for all $m$.

Let $p: F \rightarrow M$ be a connected cover of a complex $M$ having finite 1-skeleton, with Galois group $C$.

Proposition 2.1. If $C$ is finite, then

$$
p^{*}\left(\mathcal{V}_{m}^{i}(M, \mathbb{K})\right) \subseteq \mathcal{V}_{m}^{i}(F, \mathbb{K}) \quad \text { and } \quad p^{*}\left(\mathcal{R}_{m}^{i}(M, \mathbb{K})\right) \subseteq \mathcal{R}_{m}^{i}(F, \mathbb{K}),
$$

for all $i \geq 0$ and $m>0$.

Proof. Let $\mathcal{L}$ be an arbitrary right $\pi_{1}(M)$-module. In the Hochschild-Serre spectral sequence of an arbitrary connected Galois cover of a CW-complex, $p: F \rightarrow M$,

$$
E_{s t}^{2}=H_{s}\left(C, H_{t}\left(F, p^{*} \mathcal{L}\right)\right) \Rightarrow H_{s+t}(M, \mathcal{L}),
$$

see e.g. Brown's book [6, Proposition 5.6 on page 170 and Theorem 6.3 on page 171]. When $C$ is finite and $\mathcal{L}$ is a $\mathbb{K}$-representation in characteristic zero, $E_{s t}^{2}=0$, for all $s>0$ and $t \geq 0$, and the spectral sequence collapses to the isomorphism $H_{\bullet}(M, \mathcal{L})=H_{\bullet}\left(F, p^{*} \mathcal{L}\right)_{C}$, where $(\cdot)_{C}$ denotes coinvariants. By duality, we obtain an injection, $p^{\bullet}: H^{\bullet}(M, \mathcal{L}) \hookrightarrow H^{\bullet}\left(F, p^{*} \mathcal{L}\right)$, for any $\mathcal{L} \in \mathbb{T}(M, \mathbb{K})$. Our claim on characteristic varieties follows.

In the particular case when the character (local system) $\mathcal{L}$ is trivial, we may identify the induced algebra map in cohomology, $p^{\bullet}: H^{\bullet}(M, \mathbb{K}) \rightarrow H^{\bullet}(F, \mathbb{K})$, with the inclusion of fixed points,

$$
p^{\bullet}: H^{\bullet}(F, \mathbb{K})^{C} \hookrightarrow H^{\bullet}(F, \mathbb{K}) .
$$

For $\alpha \in H^{1}(F, \mathbb{K})^{C}$, the monodromy $C$-action on $H^{\bullet}(F, \mathbb{K})$ clearly gives rise to a $C$-action on the chain complex $\left(H^{\bullet}(F, \mathbb{K}), \mu_{\alpha}\right)$, with fixed subcomplex $\left(H^{\bullet}(M, \mathbb{K}), \mu_{\alpha}\right)$. Since $C$ is finite and char $\mathbb{K}=0$, we obtain an inclusion, $H^{*}\left(H^{\bullet}(M, \mathbb{K}), \mu_{\alpha}\right) \hookrightarrow H^{*}\left(H^{\bullet}(F, \mathbb{K}), \mu_{\alpha}\right)$. This proves our claim on resonance varieties.

We continue by examining the algebraic monodromy action, and the implications of its triviality on cohomology jump loci. We keep the hypotheses from Proposition 2.1.

Corollary 2.2. If $C$ acts trivially on $H^{i}(F, \mathbb{K})$, for $i \leq q$, where $q \geq 1$, then $p^{*}: H^{*}(M, \mathbb{K}) \rightarrow H^{*}(F, \mathbb{K})$ is an isomorphism for $* \leq q$ and a monomorphism for $*=q+1$. In degree $*=1$, $p^{*}$ identifies $\mathcal{R}_{m}^{i}(M, \mathbb{K})$ with $\mathcal{R}_{m}^{i}(F, \mathbb{K})$, for all $i \leq q$ and $m>0$.

Proof. Everything follows from identification (2.3). 
To obtain a similar result for characteristic varieties, we need to discuss first partial formality (in the sense from [23]). This notion can be naturally extracted from Sullivan's foundational work in rational homotopy theory [35] (see also Morgan [25]). Sullivan associates to a space $X$, in a natural way, a $\mathbb{K}$-DGA, $\Omega_{P L}^{*}(X, \mathbb{K})$; see also Bousfield and Gugenheim [4]. This is an analog of the usual de Rham DGA of a manifold. He proves that $H^{\bullet} \Omega_{P L}^{*}(X, \mathbb{K})=H^{\bullet}(X, \mathbb{K})$, as graded $\mathbb{K}$-algebras.

Sullivan goes on defining, for each $1 \leq q \leq \infty$, an important class of $\mathbb{K}$ DGA's: the $q$-minimal ones (simply called minimal, for $q=\infty$ ). A DGA map, $f$ : $\left(A^{*}, d_{A}\right) \rightarrow\left(B^{*}, d_{B}\right)$, is a $q$-equivalence (or, quasi-isomorphism, for $\left.q=\infty\right)$ if the induced map in cohomology is an isomorphism up to degree $q$, and an injection in degree $q+1$. Given any homologically connected DGA $\left(A^{*}, d_{A}\right)$ (i.e., such that $\left.H^{0}\left(A, d_{A}\right)=\mathbb{K}\right)$, he shows that there is a $q$-minimal DGA, $\left(\mathcal{M}_{q}, d\right)$, together with a $q$-equivalence, $\varphi:\left(\mathcal{M}_{q}, d\right) \rightarrow\left(A, d_{A}\right)$. Moreover, these two conditions uniquely determine $\left(\mathcal{M}_{q}, d\right)$, up to DGA isomorphism. The DGA $\left(\mathcal{M}_{q}, d\right)$ is called the $q$ minimal model of $\left(A, d_{A}\right)$ (simply the minimal model, for $q=\infty$ ), and will be denoted by $\mathcal{M}_{q}\left(A, d_{A}\right)$ (respectively by $\mathcal{M}\left(A, d_{A}\right)$, for $\left.q=\infty\right)$. For $1 \leq r \leq$ $q \leq \infty, \mathcal{M}_{r}\left(A, d_{A}\right)$ is the sub-DGA of $\mathcal{M}_{q}\left(A, d_{A}\right)$ generated by the elements of degree at most $r$.

Sullivan calls a homologically connected $\mathbb{K}$-DGA $\left(A, d_{A}\right)$ formal if $\mathcal{M}\left(A^{*}, d_{A}\right)=$ $\mathcal{M}\left(H^{*}\left(A, d_{A}\right), d=0\right)$, up to DGA isomorphism. A path-connected space $X$ is formal (over $\mathbb{K}$ ) if the DGA $\Omega_{P L}^{*}(X, \mathbb{K})$ is formal. Since the appearance of [10], where the authors prove the formality of compact Kahler manifolds, this notion played a key role in rational homotopy theory and its applications. The following notion of partial formality was investigated by Macinic in [23].

Definition 2.3. A $\mathbb{K}$-DGA $\left(A^{*}, d_{A}\right)$ with $H^{0}\left(A, d_{A}\right)=\mathbb{K}$ is $q$-formal $(1 \leq q \leq$ $\infty)$ if $\mathcal{M}_{q}\left(A^{*}, d_{A}\right)=\mathcal{M}_{q}\left(H^{*}\left(A, d_{A}\right), d=0\right)$, up to DGA isomorphism. A pathconnected space $X$ is $q$-formal (over $\mathbb{K}$ ) if the DGA $\Omega_{P L}^{*}(X, \mathbb{K})$ is $q$-formal.

Remark 2.4. A couple of useful simple properties follow directly from Definition 2.3 , via the discussion preceding it. $X$ is $\infty$-formal if and only if $X$ is formal in the sense of Sullivan. For $1 \leq r \leq q \leq \infty, q$-formality implies $r$-formality. If $p: F \rightarrow M$ is a continuous map between path-connected spaces, inducing in $\mathbb{K}$ cohomology an isomorphism up de degre $q$, and a monomorphism in degree $q+1$, then $F$ is $q$-formal if and only if $M$ is $q$-formal, over $\mathbb{K}$. Taking $M$ to be a point, it follows that $F$ is $q$-formal, if $b_{i}(F)=0$, for $i \leq q$. See also the survey [29] for more details on partial formality.

Corollary 2.5. Let $p: F \rightarrow M$ be a finite Galois cover, as in Proposition 2.1. Assume that $C$ acts trivially on $H^{i}(F, \mathbb{K})$, for $i \leq q(q \geq 1)$. Then $F$ is $q$-formal if and only if $M$ is $q$-formal, over $\mathbb{K}$.

Proof. Combine Corollary 2.2 and Remark 2.4.

Corollary 2.6. Let $p: F \rightarrow M$ be the cyclic Galois cover associated to a hyperplane arrangement in $\mathbb{P}^{n}(n \geq 2)$. If $h^{*}: H^{*}(F) \rightarrow H^{*}(F)$ is the identity, for $*<n$, then the Milnor fiber $F$ is a formal space. 
Proof. By Corollary 2.5, $F$ is $(n-1)$-formal. Up to homotopy, $F$ is a CW-complex of dimension at most $n$. These two facts together imply the formality of $F$; see [23].

Let $p: X \rightarrow K(G, 1)$ be the classifying map of a connected complex with fundamental group $G=\pi_{1}(X)$. By Remark 2.4, $X$ is 1 -formal if and only if $K(G, 1)$ is 1 -formal. When this happens, we simply say that the group $G$ is 1 formal. It is implicit in [35] that a finitely generated group $G$ is 1-formal if and only if its Malcev Lie algebra (in the sense of Quillen [31]) is a quadratic complete Lie algebra.

The 1-formality property has many interesting consequences. At the level of cohomology jump loci, these may be described as follows. Let $X$ be a connected CW-complex with finite 1-skeleton. For each $m>0$, denote by $\breve{\mathcal{V}}_{m}(X) \subseteq \mathbb{T}^{0}(X)$ the union of all non-translated irreducible components of $\mathcal{V}_{m}(X)$. Let exp: $H^{1}(X) \rightarrow$ $\mathbb{T}^{0}(X)$ be the exponential map.

Theorem 2.7 ([18]). If $X$ is 1-formal, then $\mathcal{R}_{m}(X)$ is a finite union of linear subspaces of $H^{1}(X)$, defined over $\mathbb{Q}$, and $\breve{V}_{m}(X)=\exp \left(\mathcal{R}_{m}(X)\right)$, for all $m>0$. More precisely, if $\left\{U_{i}\right\}$ are the irreducible components of $\mathcal{R}_{m}(X)$, then $\left\{\exp \left(U_{i}\right)\right\}$ are the irreducible components of $\breve{\mathcal{V}}_{m}(X)$.

Theorem 2.8. Let $p: F \rightarrow M$ be a connected, finite Galois cover with group $C$, where $M$ is a $C W$-complex with finite 1-skeleton. Assume that $C$ acts trivially on $H^{1}(F)$, and $M$ is 1-formal. Then the following hold.

The morphism $p^{*}: \mathbb{T}^{0}(M) \rightarrow \mathbb{T}^{0}(F)$ is a surjection with finite kernel. The space $F$ is 1-formal. The irreducible components of $\breve{V}_{m}(F)$ are $\left\{p^{*}(W)\right\}$, where $W$ runs through the irreducible components of $\breve{\mathcal{V}}_{m}(M)$, for all $m>0$.

Proof. The first assertion follows from the fact that $p^{*}: H^{1}(M) \rightarrow H^{1}(F)$ is an isomorphism. The second claim is implied by Corollary 2.2, via Remark 2.4. Again by Corollary 2.2, the isomorphism $p^{*}: H^{1}(M) \rightarrow H^{1}(F)$ identifies the irreducible components of $\mathcal{R}_{m}(F)$ with those of $\mathcal{R}_{m}(M)$. Let $\left\{U_{i}\right\}$ be the irreducible components of $\mathcal{R}_{m}(M)$. We infer from Theorem 2.7 that $\left\{\exp \left(U_{i}\right)\right\}$ are the irreducible components of $\breve{\mathcal{V}}_{m}(M)$, and $\left\{\exp \circ p^{*}\left(U_{i}\right)\right\}=\left\{p^{*} \circ \exp \left(U_{i}\right)\right\}$ are those of $\breve{\mathcal{V}}_{m}(F)$, which proves the last claim.

We point out that the triviality assumption on the algebraic monodromy from Corollary 2.5 is necessary, since in general the formality properties of the base space are not inherited by the total space of a finite Galois cover. The following simple example arose from conversations with Alex Suciu.

Example 2.9. Let $\mathbb{F}$ be the free group generated by $x$ and $y$. Let $K$ be the quotient of $\mathbb{F}$ by 3-fold commutators. Let $\varphi$ be the order 2 automorphism of $K$ induced by $x \mapsto x^{-1}, y \mapsto y^{-1}$. Using $\varphi$, we may construct the semidirect product $G=$ $K \rtimes(\mathbb{Z} / 2 \mathbb{Z})$. Let $M$ be the connected, finite 2-complex associate to a presentation of $G$. Consider the 2-cover, $p: F \rightarrow M$, with $\pi_{1}(F)=K$. Clearly, $h^{*}=-$ id 
on $H^{1}(F)$. Consequently, $b_{1}(M)=0$. By Remark 2.4, $M$ is 1-formal. Hence, $M$ is formal, see [23]. On the other hand, $K$ (hence $F$ ) is not 1-formal, as noticed by Morgan in [25].

Question 2.10. Is the Milnor fiber of an arbitrary hyperplane arrangement 1-formal? (Note that smooth affine varieties need not be 1-formal; see [17, Proposition 7.2].) Hugues Zuber has used our Theorem 4.1 in order to give the first example of a complex line arrangement in $\mathbb{P}^{2}$ whose Milnor fiber is not 1-formal, see [37].

\section{3. 'Exceptional' arrangements}

Let $\mathcal{A}=\left\{L_{1}, \ldots, L_{d}\right\}$ be a line arrangement in $\mathbb{P}^{2}$, with associated $d$-fold cyclic Galois cover $p: F \rightarrow M$. Let $\mathcal{A}_{1} \cup \ldots \cup \mathcal{A}_{k}$ be a partition of the set $\{1,2, \ldots, d\}$ into $k \geq 3$ subsets of the same cardinality $e>0$. Let $f_{i}=0$ be an equation for the line $L_{i}$ for $i=1, \ldots, d$, and set $Q_{j}=\prod_{i \in \mathcal{A}_{j}} f_{i}$, for $j=1, \ldots, k$. Clearly, $Q=Q_{1} \cdots Q_{k}$ is the defining polynomial of $\mathcal{A}$.

Theorem 3.1. With the above notation, assume that the vector space $\left\langle Q_{1}, \ldots, Q_{k}\right\rangle$ of degree e homogeneous polynomials has dimension 2. Then the following hold.

(i) $h^{*}: H^{1}(F) \rightarrow H^{1}(F)$ is not the identity; more precisely, the eigenspace $H^{1}(F)_{\lambda}$ has dimension at least $k-2$, for any $\lambda$ with $\lambda^{k}=1$.

(ii) Assume in addition that the pencil $f: M \rightarrow S$ given by $f=\left(Q_{1}, Q_{2}\right)$, (where $S$ is obtained from $\mathbb{P}^{1}$ by deleting $k$ points) has a connected generic fiber. Let $I=f^{*}\left(H^{1}(S)\right)$ be the corresponding maximal isotropic subspace in $H^{1}(M)$ (with respect to the cup product). Then $\operatorname{dim} I=k-1$ and there is an admissible morphism (in the sense of [1]), $f^{\prime}: F \rightarrow S^{\prime}$, where $\chi\left(S^{\prime}\right)<0$, such that the corresponding subspace $J=f^{*}\left(H^{1}\left(S^{\prime}\right)\right)$ in $H^{1}(F)$ has dimension at least $(k-1)^{2}$ and satisfies $J \cap p^{*}\left(H^{1}(M)\right)=p^{*}(I)$.

Proof. To prove (i), consider the pencil $f: M \rightarrow S$ given by $f=\left(Q_{1}, Q_{2}\right)$. Let $B=\left\{b_{1}, \ldots, b_{k}\right\}$ be the finite set such that $S=\mathbb{P}^{1} \backslash B$. By the Stein factorization, there is a finite map $p_{0}: S_{0} \rightarrow S$ and a morphism $f_{0}: M \rightarrow S_{0}$ such that the generic fiber of $f_{0}$ is connected, and $f=p_{0} \circ f_{0}$. Note that $S_{0}$ is a non-compact curve, and $p_{0}^{*}: H^{1}(S) \rightarrow H^{1}\left(S_{0}\right)$ is injective by [18, Lemma 6.10]. It follows that

$$
\chi\left(S_{0}\right) \leq \chi(S)=2-k \leq-1 .
$$

By Arapura's work, see $[1,13]$, it follows that $W=f_{0}^{*}\left(\mathbb{T}\left(S_{0}\right)\right)$ is an irreducible component of $\mathcal{V}_{1}(M)$ and that for any $\mathcal{L} \in W$ one has

$$
\operatorname{dim} H^{1}(M, \mathcal{L}) \geq \operatorname{dim} W-1=b_{1}\left(S_{0}\right)-1 \geq b_{1}(S)-1=k-2 .
$$

Note also that $f^{*}(\mathbb{T}(S)) \subset W$. 
On the other hand, a local system $\mathcal{L}^{\prime} \in \mathbb{T}(S)$ is determined by a family of complex numbers

$$
\left(\lambda_{1}, \ldots, \lambda_{k}\right) \in\left(\mathbb{C}^{*}\right)^{k}
$$

satisfying $\lambda_{1} \cdot \ldots \cdot \lambda_{k}=1$, where $\lambda_{j}$ is the monodromy of the local system $\mathcal{L}^{\prime}$ about the point $b_{j}$. For any $\lambda \in \mathbb{C}$ satisfying $\lambda^{k}=1$, we denote by $\mathcal{L}_{\lambda}^{\prime}$ the local system in $\mathbb{T}(S)$ corresponding to the choice

$$
\lambda_{1}=\ldots=\lambda_{k}=\lambda .
$$

The pull-back local system $\mathcal{L}_{\lambda}=f^{*} \mathcal{L}_{\lambda}^{\prime}$ is the unique local system on $M$ whose monodromy about each line is $\lambda$. By $[9,12]$ we know that

$$
\operatorname{dim} H^{1}(F)_{\lambda}=\operatorname{dim} H^{1}\left(M, \mathcal{L}_{\lambda}\right) .
$$

This dimension is at least $k-2$ since $\mathcal{L}_{\lambda} \in W$, which proves claim (i).

To prove claim (ii), we proceed as follows. For $j=3, \ldots, k$ there are unique complex numbers $\alpha_{j}, \beta_{j}$ such that $Q_{j}=\alpha_{j} Q_{1}+\beta_{j} Q_{2}$. Consider the homogeneous polynomial $G(u, v)=u v \prod_{j=3, k}\left(\alpha_{j} u+\beta_{j} v\right)$. Let $S=M(G)$ and $H=F(G)$ be the corresponding complement in $\mathbb{P}^{1}$ and Milnor fiber in $\mathbb{C}^{2}$.

The map $g: F \rightarrow H, g(x, y, z)=\left(Q_{1}(x, y, z), Q_{2}(x, y, z)\right)$ is well-defined (since $Q=Q_{1} \cdot \ldots \cdot Q_{k}$ ) and surjective. If the generic fiber of $g$ is connected, then we take $S^{\prime}=H$ and $f^{\prime}=g$. Otherwise, there is a Stein factorization $g=p^{\prime} \circ f^{\prime}$, where $p^{\prime}: S^{\prime} \rightarrow H$ is a finite morphism and $f^{\prime}: F \rightarrow S^{\prime}$ has connected generic fibers. Note that both $p^{\prime}$ and $f^{\prime}$ induce monomorphisms in cohomology, in degree 1, by [18, Lemma 6.10]. Using the same argument as in Part (i), we may infer that $\chi\left(S^{\prime}\right) \leq \chi(H)<0$.

To show that $J \cap p^{*}\left(H^{1}(M)\right)=p^{*}(I)$, use the obvious inclusion $J \cap$ $p^{*}\left(H^{1}(M)\right) \supseteq p^{*}(I)$, and the fact that $I$ is a maximal isotropic subspace in $H^{1}(M)$, see [14]. Finally, $\operatorname{dim} J=\operatorname{dim} H^{1}\left(S^{\prime}\right) \geq \operatorname{dim} H^{1}(H)=(k-1)^{2}$. This completes the proof.

Corollary 3.2. Under the assumptions from Theorem 3.1 (ii), there exist components, $W \subseteq \mathcal{V}_{1}(M)$ and $W^{\prime} \subseteq \mathcal{V}_{1}(F)$, both non-translated, with $\operatorname{dim} W^{\prime}>\operatorname{dim} W$, whose tangent spaces at 1 satisfy $p^{*}\left(T_{1} W\right)=T_{1} W^{\prime} \cap p^{*}\left(H^{1}(M)\right)$. In particular, the monodromy action on $H^{1}(F)$ is non-trivial.

Proof. Consider the admissible maps, $f: M \rightarrow S$ and $f^{\prime}: F \rightarrow S^{\prime}$, from Theorem 3.1 (ii). Since $\chi(S), \chi\left(S^{\prime}\right)<0$, we obtain non-translated components, $W=f^{*}(\mathbb{T}(S))$ and $W^{\prime}=f^{\prime *}\left(\mathbb{T}\left(S^{\prime}\right)\right)$, by Arapura theory [1]. Clearly, $T_{1} W=I$ and $T_{1} W^{\prime}=J$, using the notation from Theorem 3.1. All claims except the last (on monodromy) follow from Theorem 3.1 (ii). The triviality of $h^{*}: H^{1}(F) \rightarrow H^{1}(F)$ would imply that $p^{*}(W)$ is an irreducible component of $\mathcal{V}_{1}(F)$, due to Theorem 2.8. This contradicts the strict inclusion $p^{*}(W) \subset W^{\prime}$.

Corollary 3.3. The admissible map $f^{\prime}: F \rightarrow S^{\prime}$ from Theorem 3.1 (ii) is not a rational pencil, i.e., $S^{\prime}$ is not an open subset of $\mathbb{P}^{1}$. 
Proof. Consider the induced map on compactifications, $\widehat{p^{\prime}}: \widehat{S^{\prime}} \rightarrow \widehat{H}$, to infer that $b_{1}\left(\widehat{S^{\prime}}\right) \geq b_{1}(\widehat{H})=(k-1)(k-2)>0$.

Remark 3.4. (i) At this point, it seems worth recalling a couple of relevant facts from [19]. Firstly, admissible maps may be constructed from arbitrary multinet structures, in the following way. Set $Q_{j}=\prod_{i \in \mathcal{A}_{j}} f_{i}^{\mu(i)}$, for $j=1, \ldots, k$. Then the subspace $\left\langle Q_{1}, \ldots, Q_{k}\right\rangle$ is 2-dimensional, and the associated map, $f=\left(Q_{1}, Q_{2}\right)$ : $M \rightarrow S=\mathbb{P}^{1} \backslash\{k$ points $\}$, has connected generic fiber, as needed in Theorem 3.1. Secondly, a degree $e$ pencil with connected fibers (as defined in [19]), having $k \geq 3$ completely reducible fibers, $Q_{j}=\prod f_{i}^{\mu(i)}$ (where all factors $f_{i}$ have degree 1 ), for $j=1, \ldots, k$, gives rise to a multinet structure with multiplicity function $\mu$, on the arrangement consisting of the lines $\left\{f_{i}=0\right\}$. Finally, every multinet arises in this way. Clearly, the reduced multinets correspond to the case when all special fibers are reduced. When such a reduced structure exists, $h^{*} \neq \mathrm{id}$, on $H^{1}(F)$, by Corollary 3.2. The existence of a reduced multinet also implies that the characteristic varieties of the Milnor fiber $F$ are more subtle than those of a line arrangement complement, which are known to come from rational pencils; see Corollary 3.3.

(ii) If only non-reduced multinet structures exist, the monodromy action on $H^{1}(F)$ may well be trivial. Consider the $B_{3}$-arrangement, whose defining equation is given by $Q=x y z\left(x^{2}-y^{2}\right)\left(y^{2}-z^{2}\right)\left(z^{2}-x^{2}\right)$. The pencil

$$
\left\langle Q_{1}=x^{2}\left(y^{2}-z^{2}\right), Q_{2}=y^{2}\left(z^{2}-x^{2}\right), Q_{3}=z^{2}\left(x^{2}-y^{2}\right)\right\rangle
$$

gives rise to a multinet on $B_{3}$, with multiplicities equal to 2 on $x, y$ and $z$, and equal to 1, otherwise. See [19, Example 3.6]. As shown in [9], the monodromy action on $H^{1}(F)$ is trivial.

(iii) We point out that the non-triviality of the monodromy action on $H^{1}(F)$ may be deduced from the existence of a non-reduced multinet structure, provided its multiplicity function $\mu$ enjoys some special properties. Let us examine the family of line arrangements $\mathcal{A}_{r}$ from [19, Example 4.6], having defining polynomial

$$
x y z\left(x^{r}-y^{r}\right)\left(y^{r}-z^{r}\right)\left(z^{r}-x^{r}\right), \quad \text { and } \quad d=3(r+1) .
$$

The corresponding pencil is

$$
\left\langle Q_{1}=x^{r}\left(y^{r}-z^{r}\right), Q_{2}=y^{r}\left(z^{r}-x^{r}\right), Q_{3}=z^{r}\left(x^{r}-y^{r}\right)\right\rangle,
$$

and the multiplicity function takes the value $r$ on on $x, y$ and $z$, and 1 , otherwise. Assume that $r \equiv 1$ (modulo 3). Let $\lambda$ be a primitive root of unity of order 3 . Then the local system $\mathcal{L}_{\lambda}$ constructed as in the proof of Theorem 3.1 (i) has monodromy $\lambda$ about every line of $\mathcal{A}_{r}$ (since $\lambda^{r}=\lambda$ ). Consequently, the same argument as in Theorem 3.1 (i) implies that the eigenspace $H^{1}(F)_{\lambda}$ has dimension at least 1 .

(iv) The inequality $\operatorname{dim} H^{1}(F)_{\lambda} \geq k-2$ from Theorem 3.1 (i) above is shown to be an equality under some additional condition in [7, Theorem 1 (ii)]. 
Example 3.5. For the $A_{3}$-arrangement and the Pappus configuration $\left(9_{3}\right)_{1}$, it follows from [9] that the inequality in Theorem 3.1 (i) is in fact an equality. This is the case whenever the generic fiber of $g: F \rightarrow H$ is connected, i.e. $f^{\prime}=g$, and the local system $\mathcal{L}_{\lambda}$ is 1-admissible, which is equivalent to $\operatorname{dim} H^{1}\left(M, \mathcal{L}_{\lambda}\right)=\operatorname{dim} W-1$, see [16] for this equivalence.

Example 3.6. Consider the Hesse arrangement consisting of the 12 lines that occur in the 4 special fibers of the Hesse pencil $f=\left(x^{3}+y^{3}+z^{3}, x y z\right)$; see [19]. Here $d=12$, and there is a partition with $k=4$. Theorem 3.1 implies that in this case $H^{1}(F)_{-1}, H^{1}(F)_{i}$ and $H^{1}(F)_{-i}$ have each dimension at least 2. Using [7, Theorem 1 (ii)], one finds that all these dimensions are equal to 2 .

\section{Monodromy action and mixed Hodge structure}

We begin this section with a general result on Milnor fibers, valid for a class of polynomials that includes the case of line arrangements. Let $Q \in \mathbb{C}\left[x_{0}, \ldots, x_{n}\right]$, $n \geq 2$, be a homogeneous polynomial of degree $d$ defining a hypersurface $V(Q) \subset$ $\mathbb{P}^{n}$ having only isolated singularities. Let $F=F(Q)$ (respectively $M=M(Q)$ ) be the corresponding Milnor fiber (respectively complement), and let $p: F \rightarrow M$ be the canonical projection. The rational cohomology $H^{*}(F, \mathbb{Q})$ has a natural direct sum decomposition

$$
H^{*}(F, \mathbb{Q})=H^{*}(F, \mathbb{Q})_{1} \oplus H^{*}(F, \mathbb{Q})_{\neq 1}
$$

where $H^{*}(F, \mathbb{Q})_{1}=\operatorname{ker}\left(h^{*}-1\right)=p^{*} H^{*}(M, \mathbb{Q})$ is the eigenspace corresponding to the eigenvalue $\lambda=1$ of the monodromy operator $h^{*}: H^{*}(F, \mathbb{Q}) \rightarrow H^{*}(F, \mathbb{Q})$, and $H^{*}(F, \mathbb{Q})_{\neq 1}=\operatorname{ker}\left(\left(h^{*}\right)^{d-1}+\ldots+1\right)$. A different approach to the next result in the case of line arrangements can be found in $[7,(2.5 .3)]$.

Theorem 4.1. With the above notation, the mixed Hodge structure on $H^{n-1}(F, \mathbb{Q})$ is split, i.e., the subspaces $H^{n-1}(F, \mathbb{Q})_{1}$ and $H^{n-1}(F, \mathbb{Q})_{\neq 1}$ inherit pure Hodge structure from $H^{n-1}(F, \mathbb{Q})$, such that $H^{n-1}(F, \mathbb{Q})_{1}\left(\right.$ respectively $H^{n-1}(F, \mathbb{Q})_{\neq 1}$ ) has weight $n$ (respectively $n-1)$.

Proof. To study the MHS on $H^{*}(M)$, one may use the following exact sequence of MHS, see [30, page 138]

$$
\ldots \rightarrow H^{n}(V(Q)) \rightarrow H_{c}^{n+1}(M) \rightarrow H^{n+1}\left(\mathbb{P}^{n}\right) \rightarrow \ldots
$$

Since $V(Q)$ has only isolated singularities, it follows that $H^{n}(V(Q))$ is pure of weight $n$, see [33]. Moreover, the morphism $H^{n+1}\left(\mathbb{P}^{n}\right) \rightarrow H^{n+1}(V(Q))$ is an isomorphism, being a monomorphism and the two spaces having the same dimension: 0 for $n$ even and 1 for $n$ odd. Hence $H_{c}^{n+1}(M)$ is pure of weight $n$. Using the duality between $H^{*}(M)$ and $H_{c}^{*}(M)$, see [30, page 155], it follows that $H^{n-1}(M)$ is pure of weight $n$. 
Let $\bar{F}$ be the projective hypersurface in $\mathbb{P}^{n+1}$ defined by $\bar{Q}(x, t)=Q(x)+t^{d}=0$. It is known that $\operatorname{dim} H^{n-1}(F)_{\neq 1}=\operatorname{dim} H^{n}(M(\bar{Q}))=\operatorname{dim} \operatorname{coker}\left(H^{n+1}\left(\mathbb{P}^{n+1}\right) \rightarrow\right.$ $H^{n+1}(V(\bar{Q}))$, see [11, page 196 and 206].

Since $\bar{F}$ is a compactification of $F$ such that $\bar{F} \backslash F=V(Q)$, we get the exact sequence of MHS

$$
\ldots \rightarrow H^{n}(V(Q)) \rightarrow H_{c}^{n+1}(F) \rightarrow H^{n+1}(V(\bar{Q})) \rightarrow H^{n+1}(V(Q)) \rightarrow \ldots
$$

The fact that $H^{n}(V(Q))$ (respectively $H^{n+1}(V(\bar{Q}))$ ) is pure of weight $n$ (respectively $n+1)$, implies that $G r_{n+1}^{W} H_{c}^{n+1}(F)$ has the same dimension as $\operatorname{dim} H^{n-1}(F)_{\neq 1}$. To see this, note that the morphism

$$
H^{n+1}\left(\mathbb{P}^{n+1}\right) \rightarrow H^{n+1}(V(\bar{Q}))
$$

is injective, while the morphism

$$
H^{n+1}(V(\bar{Q})) \rightarrow H^{n+1}(V(Q))
$$

is surjective, and $\operatorname{dim} H^{n+1}\left(\mathbb{P}^{n+1}\right)=\operatorname{dim} H^{n+1}(V(Q))$. It follows that the dimension of the cokernel of the first morphism, which is $\operatorname{dim} H^{n-1}(F)_{\neq 1}$ as seen above, coincides with the dimension of the kernel of the second morphism, which is precisely the dimension of $G r_{n+1}^{W} H_{c}^{n+1}(F)$ by (4.2).

By the duality between $H^{n-1}(F)$ and $H_{c}^{n+1}(F)$, we see that

$$
\operatorname{dim} W_{n-1} H^{n-1}(F)=\operatorname{dim} H^{n-1}(F)_{\neq 1} .
$$

Now, the equality $H^{n-1}(F, \mathbb{Q})_{1}=p^{*} H^{n-1}(M, \mathbb{Q})$ and our proof above show that $H^{n-1}(F, \mathbb{Q})_{1}$ is a pure HS of weight $n$.

Consider now the subspace $E=W_{n-1} H^{n-1}(F, \mathbb{Q})$. Then $E$ is $h^{*}$ invariant, $E \cap H^{n-1}(F, \mathbb{Q})_{1}=0$ and $\operatorname{dim} E=\operatorname{dim} H^{n-1}(F, \mathbb{Q})_{\neq 1}$. These three properties imply that $E=H^{n-1}(F)_{\neq 1}$. Since the proof above implies also that $W_{n-2} H^{n-1}(F, \mathbb{Q})=W_{n+2} H_{c}^{n+1}(F, \mathbb{Q})=0$, all the claims are proven.

As an application, we offer a converse to Corollary 2.2, for $q=1$.

Corollary 4.2. Let $p: F \rightarrow M$ be the Galois cover associated to an arbitrary line arrangement. The algebraic monodromy action on $H^{1}(F)$ is trivial if and only if $p^{*}\left(\mathcal{R}_{1}(M)\right)=\mathcal{R}_{1}(F)$.

Proof. We will show that $\mathcal{R}_{1}(F) \nsubseteq p^{*}\left(\mathcal{R}_{1}(M)\right)$, if $h^{*} \neq$ id on $H^{1}(F)$. Indeed, we know from Theorem 4.1 that $W_{1} H^{1}(F)=\bigoplus_{\lambda \neq 1} H^{1}(F)_{\lambda} \neq 0$. Let us first assume that $H^{1}(F)_{\lambda} \neq 0$, for some $\lambda \neq \pm 1$. Pick linearly independent eigenvectors of $h^{*}, \alpha \in H^{1}(F)_{\lambda}$ and $\beta \in H^{1}(F)_{\lambda^{-1}}$. We will conclude in this case by showing that $\alpha \cup \beta=0$. Clearly, $\alpha \cup \beta \in W_{2} H^{2}(F) \cap p^{*}\left(H^{2}(M)\right)=p^{*}\left(W_{2} H^{2}(M)\right)$. Since the MHS on $H^{2}(M)$ is pure of type $(2,2)$, by [32], $W_{2} H^{2}(M)=0$, and we are done. In the remaining case, $W_{1} H^{1}(F)=H^{1}(F)_{-1} \neq 0$. Since this space has even dimension, we may find two linearly independent eigenvectors of $h^{*}, \alpha, \beta \in H^{1}(F)_{-1}$. Then $\alpha \cup \beta \in p^{*}\left(W_{2} H^{2}(M)\right)=0$, as before. 
We close this section with an addendum to Theorem 2.8, concerning translated components. Let $M=M(Q)$ be a curve complement in $\mathbb{P}^{2}$, with associated cyclic Galois cover $p: F \rightarrow M$. Assume $h^{*}=\mathrm{id}$ on $H^{1}(F)$.

We need to briefly recall the general theory, following [1] and [13]. Let $X$ be a smooth, irreducible, quasi-projective variety. The positive-dimensional components, $W^{\prime}$, of $\mathcal{V}_{1}(X)$, are intimately related to admissible maps, that is, surjective regular maps onto smooth curves, $f: X \rightarrow S$, having connected generic fiber. Each $W^{\prime}$ is a (possibly translated) subtorus of the form $\rho f^{*}(\mathbb{T}(S))$, where $f$ is admissible, $\chi(S) \leq 0$, and $\rho \in \mathbb{T}(X)$ has finite order. The number of components with the same direction, $W:=f^{*}(\mathbb{T}(S))$, denoted by $n(W)$, is computable in terms of the multiplicities of the fibers of $f$. For $c \in S$, we denote the corresponding multiplicity by $m_{c}(f)$.

A key result due to Arapura, [1, Proposition V.1.7], guarantees that $f^{*}(\mathbb{T}(S))$ is a component of $\mathcal{V}_{1}(X)$, whenever $f$ is admissible and $\chi(S)<0$. We will need the following converse implication.

Proposition 4.3. Let $f: X \rightarrow S$ be a surjective regular map. If $\chi(S)<0$ and $f^{*}(\mathbb{T}(S))$ is an irreducible component of $\mathcal{V}_{1}(X)$, then $f$ is admissible.

Proof. Take a Stein factorisation, $f=q \circ f^{\prime}$, where $f^{\prime}: X \rightarrow S^{\prime}$ is admissible, and $q: S^{\prime} \rightarrow S$ is finite. Now, we will make an estimate for Euler numbers, valid for an arbitrary finite map $q$ between smooth, irreducible curves.

Let $e$ be the degree of $q$. Let $S=\cup_{k=1, e} S_{k}$ be the canonical partition: each $S_{k}$ consists of those $c \in S$ having exactly $k q$-preimages. For $k<e, S_{k}$ is a finite set, of cardinality $s_{k} \geq 0$. Then $S^{\prime}=\cup_{k=1, e} q^{-1}\left(S_{k}\right)$, and the restriction $q: q^{-1}\left(S_{e}\right) \rightarrow S_{e}$ is a non-ramified $e$-fold cover. Using the additivity of Euler numbers with respect to constructible partitions (see [12]), we find that

$$
\chi\left(S^{\prime}\right)-\chi(S)=(e-1) \chi\left(S_{e}\right)+\sum_{1 \leq k<e}(k-1) s_{k},
$$

which leads to

$$
\chi\left(S^{\prime}\right)-\chi(S)=(e-1) \chi(S)+\sum_{1 \leq k<e}(k-e) s_{k} .
$$

We infer from (4.4) that $\chi\left(S^{\prime}\right)<0$, since $\chi(S)<0$. Therefore, $f^{\prime *}\left(\mathbb{T}\left(S^{\prime}\right)\right)$ is an irreducible component of $\mathcal{V}_{1}(X)$, containing $f^{*}(\mathbb{T}(S))$, which implies that $f^{\prime *}\left(\mathbb{T}\left(S^{\prime}\right)\right)=f^{*}(\mathbb{T}(S))$. In particular $b_{1}\left(S^{\prime}\right)=b_{1}(S)$, hence $\chi\left(S^{\prime}\right)=\chi(S)$. Since $(k-e) s_{k} \leq 0$ for any $k$, and $\chi(S)<0$, we deduce from (4.4) that $e=1$. Therefore, the generic fiber of $f$ is connected, as asserted, since $f^{\prime}$ has this property.

Going back to the case when $X=M(Q)$ is a curve complement in $\mathbb{P}^{2}$, and $h^{*}=$ id on $H^{1}(F)$, we recall from Theorem 2.8 the correspondence $W \mapsto$ $p^{*}(W)$. This gives a dimension-preserving bijection between non-translated subtori in $\mathbb{T}(M)$ and $\mathbb{T}(F)$, that identifies the non-translated irreducible components in $\mathcal{V}_{1}(M)$ and $\mathcal{V}_{1}(F)$. In geometric terms, this may be rephrased as follows. 
Corollary 4.4. Let $p: F \rightarrow M$ be the Galois cover associated to a plane projective curve. Assume the algebraic monodromy action on $H^{1}(F)$ is trivial. If $f: M \rightarrow S$ is admissible and $\chi(S)<0$, then $g:=f \circ p: F \rightarrow S$ is admissible, too.

Note also that any admissible map, $f: M \rightarrow S$ or $g: F \rightarrow S$, must be a rational pencil (compare to Corollary 3.3). This is due to the fact that $W_{1} H^{1}(M)=$ 0 , and consequently $W_{1} H^{1}(F)=0$; see [18, Proposition 7.2].

Proposition 4.5. Under the assumptions from Corollary 4.4, the following hold.

(i) The bijection $W \mapsto p^{*}(W)$ identifies, for $k>1$, the $k$-dimensional directions of irreducible components in $\mathcal{V}_{1}(M)$ and $\mathcal{V}_{1}(F)$. Moreover, $n(W)=$ $n\left(p^{*}(W)\right)$.

(ii) If $W$ is a 1-dimensional direction of irreducible component in $\mathcal{V}_{1}(M)$, then so is $p^{*}(W)$ in $\mathcal{V}_{1}(F)$, and $n(W) \leq n\left(p^{*}(W)\right)$.

Proof. (i) For $k>1$, it follows from [13] that (for both $M$ and $F$ ) the $k$-dimensional directions from our statement coincide with the non-translated $k$-dimensional components of $\mathcal{V}_{1}$. Moreover, they are all of the form $f^{*}(\mathbb{T}(S))$, where $f$ is an admissible map onto a rational curve with $\chi(S)=1-k$, and $n\left(f^{*}(\mathbb{T}(S))\right)=\prod_{c} m_{c}(f)$, where the product is taken over those $c \in S$ with $m_{c}(f)>1$. Our claims follow then from Theorem 2.8, Corollary 4.4, and the remark that $m_{c}(f \circ p)=m_{c}(f)$, for all $c \in S$, since $p$ is a submersion.

(ii) For $k=1$, we know from [13] that the 1-dimensional directions (for both $M$ and $F$ ) coincide with the subtori of the form $f^{*}\left(\mathbb{T}\left(\mathbb{C}^{*}\right)\right)$, where $f$ is an admissible map onto $\mathbb{C}^{*}$, having at least one multiple fiber. In this case, $1+$ $n\left(f^{*}\left(\mathbb{T}\left(\mathbb{C}^{*}\right)\right)\right)=\prod_{c} m_{c}(f)$. If $f: M \rightarrow \mathbb{C}^{*}$ satisfies the above conditions, let us consider the map $g:=f \circ p$.

We may factor it as in the proof of Proposition 4.3, $g=q \circ g^{\prime}$, with $g^{\prime}: F \rightarrow S^{\prime}$ admissible and $q: S^{\prime} \rightarrow \mathbb{C}^{*}$ finite. We also know from [15, Corollary 3.21] that $f^{*}\left(H^{1}\left(\mathbb{C}^{*}, \mathbb{C}\right)\right)$ is a maximal isotropic subspace in $H^{1}(M)$, which implies the same property, for $g^{*}\left(H^{1}\left(\mathbb{C}^{*}, \mathbb{C}\right)\right)$ in $H^{1}(F)$. The inclusion $g^{*}\left(H^{1}\left(\mathbb{C}^{*}, \mathbb{C}\right)\right) \subseteq$ $g^{*}\left(H^{1}\left(S^{\prime}, \mathbb{C}\right)\right)$ must then be an equality, which forces $S^{\prime}$ to be $\mathbb{C}^{*}$, and $q$ to induce a cohomology isomorphism. In this case, (4.4) becomes

$$
\sum_{k<e}(k-e) s_{k}=0,
$$

therefore $q$ is unramified. This implies that $m_{c}(f)=m_{c}\left(q \circ g^{\prime}\right)=$ g.c.d. $\left\{m_{c^{\prime}}\left(g^{\prime}\right) \mid\right.$ $\left.c^{\prime} \in q^{-1}(c)\right\}$, for all $c \in \mathbb{C}^{*}$.

We thus see that each multiple fiber $f^{-1}(c)$ gives rise to $e$ multiple fibers of $g^{\prime}$. In particular, $g^{\prime *}\left(\mathbb{T}\left(\mathbb{C}^{*}\right)\right)=g^{*} \circ q^{*}\left(\mathbb{T}\left(\mathbb{C}^{*}\right)\right)=p^{*} \circ f^{*}\left(\mathbb{T}\left(\mathbb{C}^{*}\right)\right)$ is a 1-dimensional direction in $\mathcal{V}_{1}(F)$. Clearly, $\prod_{c} m_{c}(f)^{e} \leq \prod_{c^{\prime}} m_{c^{\prime}}\left(g^{\prime}\right)$, which verifies our last claim. 


\section{References}

[1] D. ARAPURA, Geometry of cohomology support loci for local systems. I, J. Algebraic Geom. 6 (1997), 563-597.

[2] A. Beauville, Annulation du $H^{1}$ pour les fibrés en droites plats, In: "Complex Algebraic Varieties" (Bayreuth, 1990), Lect. Notes in Math., Vol. 1507, Springer, Berlin, 1992, 1-15.

[3] B. BERCEANU and S. PAPADIMA, Universal representations of braid and braid-permutation groups, J. Knot Theory Ramifications 18 (2009), 999-1019.

[4] A. K. Bousfield and V. K. A. M. Gugenheim, "On PL De Rham Theory and Rational Homotopy Type", Memoirs Amer. Math. Soc., Vol. 179, Amer. Math. Soc., Providence, RI, 1976.

[5] E. BRIESKORN, Sur les groupes de tresses, In: "Séminaire Bourbaki", 1971/72, Lect. Notes in Math. Vol. 317, Springer-Verlag, 1973, 21-44.

[6] K. S. Brown, "Cohomology of Groups", Grad. Texts in Math., Vol. 87, Springer-Verlag, New York-Berlin, 1982.

[7] N. Budur, A. DimCA and M. SAITO, First Milnor cohomology of hyperplane arrangements, Contemp. Math. 538 (2011), 279-292.

[8] A. D. R. Choudary, A. Dimca and S. Papadima, Some analogs of Zariski's theorem on nodal line arrangements, Algebr. Geom. Topol. 5 (2005), 691-711.

[9] D. C. Cohen and A. I. Suciu, On Milnor fibrations of arrangements, J. London Math. Soc. 51 (1995), 105-119.

[10] P. Deligne, P. Griffiths, J. Morgan and D. Sullivan, Real homotopy theory of Kähler manifolds, Invent. Math. 29 (1975), 245-274.

[11] A. DimcA, "Singularities and Topology of Hypersurfaces", Universitext, Springer-Verlag, 1992.

[12] A. DimCA, "Sheaves in Topology", Universitext, Springer-Verlag, 2004.

[13] A. DimCA, Characteristic varieties and constructible sheaves, Atti Accad. Naz. Lincei Cl. Sci. Fis. Mat. Natur. Rend. Lincei (9) Mat. Appl. 18 (2007), 365-389.

[14] A. DimcA, On the isotropic subspace theorems, Bull. Math. Soc. Sci. Math. Roumanie 51 (2008), 307-324.

[15] A. DimcA, Pencils of plane curves and characteristic varieties, In: "Arrangements, Local Systems and Singularities", Progress in Mathematics, Vol. 283, Birkhäuser, 2009, 59-82.

[16] A. DimCA, On admissible rank one local systems, J. Algebra, 321 (2009), 3145-3157.

[17] A. Dimca, S. PAPADIMA and A. SuciU, Quasi-Kähler groups, 3-manifold groups, and formality, Math. Z., available online DOI: 10.1007/s00209-010-0664-y

[18] A. Dimca, S. PAPADIMA and A. SUCiU, Topology and geometry of cohomology jump loci, Duke Math. J. 148 (2009), 405-457.

[19] M. FALK and S. YUZVINSKY, Multinets, resonance varieties, and pencils of plane curves, Compos. Math. 143 (2007), 1069-1088.

[20] M. GREEN and R. LAZARSFELD, Higher obstructions to deforming cohomology groups of line bundles, J. Amer. Math. Soc. 4 (1991), 87-103.

[21] M. KAPOVICH and J. Millson, On representation varieties of Artin groups, projective arrangements and the fundamental groups of smooth complex algebraic varieties, Inst. Hautes Études Sci. Publ. Math. 88 (1998), 5-95.

[22] A. LIBGOBER, Eigenvalues for the monodromy of the Milnor fibers of arrangements, In: "Trends in singularities", Trends Math., Birkhäuser, Basel, 2002, 141-150.

[23] A. MACINIC, Cohomology rings and formality properties of nilpotent groups, J. Pure Appl. Algebra 214 (2010), 1818-1826.

[24] A. MACINIC and S. PAPADIMA, On the monodromy action on Milnor fibers of graphic arrangements, Topology Appl. 156 (2009), 761-774.

[25] J. W. Morgan, The algebraic topology of smooth algebraic varieties, Inst. Hautes Études Sci. Publ. Math. 48 (1978), 137-204. 
[26] P. ORLIK and L. SOlOMON, Combinatorics and topology of complements of hyperplanes, Invent. Math. 56 (1980), 167-189.

[27] S. PAPADIMA and A. I. Suciu, Toric complexes and Artin kernels, Adv. Math. 220 (2009), 441-477.

[28] S. PAPADIMA and A. I. SUCIU, Algebraic monodromy and obstructions to formality, Forum Math. 22 (2010), 973-983.

[29] S. Papadima and A. I. Suciu, Geometric and algebraic aspects of 1-formality, Bull. Math. Soc. Sci. Math. Roumanie 52 (2009), 355-375.

[30] C. Peters and J. Steenbrink, "Mixed Hodge Structures", Ergeb. der Math. und ihrer Grenz. 3, Folge 52, Springer, 2008.

[31] D. Quillen, Rational homotopy theory, Ann. of Math. 90 (1969), 205-295.

[32] B. Z. SHAPIRO, The mixed Hodge structure of the complement to an arbitrary arrangement of affine complex hyperplanes is pure, Proc. Amer. Math. Soc. 117 (1993), 931-933.

[33] J. SteEnbrink, Mixed Hodge structures associated with isolated singularities, In: "Singularities", Part 2 (Arcata, 1981), Proc. Symp. Pure Math. 40, Amer. Math. Soc., 1983, 513-536.

[34] A. SUCIU, Fundamental groups of line arrangements: enumerative aspects, In: "Advances in Algebraic Geometry Motivated by Physics" (Lowell, MA, 2000), Contemp. Math. Vol. 276, Amer. Math. Soc., Providence, RI, 2001, 43-79.

[35] D. Sullivan, Infinitesimal computations in topology, Inst. Hautes Études Sci. Publ. Math. 47 (1977), 269-331.

[36] S. YUZVINSKY, A new bound on the number of special fibers in a pencil of curves, Proc. Amer. Math. Soc. 137 (2009), 1641-1648.

[37] H. ZUBER, Non-formality of Milnor fibres of line arrangements, Bull. London Math. Soc. 42 (2010), 905-911.

Laboratoire J. A. Dieudonné

UMR du CNRS 6621

Université de Nice Sophia-Antipolis

Parc Valrose

06108 Nice Cedex 02, France

dimca@unice.fr

Institute of Mathematics Simion Stoilow

P.O. Box 1-764

RO-014700 Bucharest, Romania

Stefan.Papadima@imar.ro 